\section{An efficient sort/search algorithm for linked linear lists}

\section{RICHARD S. LEHMAN \\ Franklin and Marshall College, Lancaster, Pennsylvania 17604}

A linked linear list is a data structure in which each data element consists of two (or more) distinct parts. One part is obviously the datum itself (a data value, a name, a syllable in a learning experiment, a pointer into a table of data on a random access device, etc.). The second segment of each entry is a pointer to the location of the next datum in the list. Logically adjacent data values need not be physically contiguous, since the pointer always will lead to the next item. The linked list is widely applicable in many programming problems; it is most frequently found in simulation programming, data base acquisition and interrogation systems, and compilers; some statistical systems make use of lists as well. Additional information about lists and their uses can be found in any standard text on data structures or in Lehman (1977).

The process by which data are arranged in an ascending or descending order is known as sorting. Locating a specific item in a set of data invokes a search process. When an array is sorted, a search must generally be employed as well to determine the location of an item to be added. Searches and sorts are complementary processes and have received a great deal of attention from commercial programmers; if poorly programmed, they can consume vast amounts of computer time if the data array is large.

When data (numbers, names, etc.) are arranged in a simple array of the kind familiar to BASIC or FORTRAN programmers, a number of sort procedures are available and generally well-known-the bubble sort, Shell sort, and so forth. These procedures arrange the contents of an array into order according to the value of the data; for example, location $i+1$ contains the next larger value after location i. Lehman (1977) presents algorithms for sorting arrays, for searching, and for handling the problems associated with additions and deletions to the data contained in arrays.

If the data structure required in a program is a linked list instead of an array, the sorting, searching, and addition/deletion problems become considerably more complex. The purpose of this paper is to call attention to a highly efficient procedure for sorting and searching in linked lists. The algorithm is related to the multiplelist insertion sort of Knuth (1973) and the sublist sort of Flores (1969).

The algorithm. Consider data stored in a list array $L$. The array holds a linked linear list in sorted order. Assume that location $L(i)$ contains a data value and that location $L(i+1)$ contains a pointer to the next larger data value. Inserting a new value into the list so that the sorted order is maintained involves a search of $\mathrm{L}$ to find the datum just smaller than the new entry. Assume that a search indicates that a new item must logically follow (is just larger than) the value in $L(j)$. If the first empty space in the array is $L(n)$, the new item is placed there. Now the pointer associated with $L(j)$ must be changed to indicate that $L(j)$ has a new successor; thus, $L(j+1)$, the pointer for $L(j)$, is set to $L(n)$. Since the item in $L(n)$ now precedes the data value that was formerly the successor of $L(j), L(n)$ 's pointer $[L(n+1)]$ must be given the value of the former pointer of $L(j)$; that is, $L(n+1)$ must have the value that was previously in $\mathbf{L}(j+1)$. These steps accomplished, the new value has been inserted into the list.

A critical step in the sort is the location of the predecessor of the new value, $L(j)$ in the example. This is a search operation and is likely to be time-consuming. The key to efficient searching in a linked list involves maintaining a separate auxiliary array of values showing where certain selected elements of the data values are stored. With this array, the search can be speeded significantly. If the data to be sorted consist of numbers selected from a rectangular distribution of the values $1-10,000$, it makes little sense to start at the beginning of the list to find the place for 7,562. If the program knew roughly where in the list the predecessor of 7,562 was likely to be, then the search could be speeded greatly.

In order to establish the auxiliary array, some information about the distribution of the entries to be sorted is required. If they follow some known form (rectangular, normal, etc.), then the problem is minimized. Otherwise, some values (perhaps 100) must be inspected and appropriate key values estimated.

Experimentation with the algorithm has indicated that three key values, representing the quartile points in the distribution of values to be sorted, are sufficient to produce sort and search speeds up to three or four times faster than a sequential search procedure during sorting. The search proceeds by locating the quartile point closest to the required value. The list location of that quartile is found in a table of pointers. The designated list element is then used as the starting point for a search. It is inspected and, if it is not the required value, its pointer is followed to the next entry, and so forth.

Implementation and availability. A set of 10 FORTRAN IV subroutines implement the algorithm and all necessary supplementary operations, such as initializing the list array, inserting new elements, and both sorting and searching on the list structure. They, along with an illustrative main driver program used for comparative 
timing tests and a detailed presentation of a numerical example, are available at no cost from the author at: Department of Psychology, Franklin and Marshall College, Lancaster, Pennsylvania 17604.

\section{REFERENCES}

Flores. I. Computer sorting. Englewood Cliffs, N.J: PrenticeHall. 1969.
KNUTH, D. E. The art of computer programming: Sorting and searching (Vol. 3). Reading, Mass: Addison-Wesley, 1973.

LEHMAN, R. S. Computer simulation and modeling. Hillsdale, N.J: Erlbaum. 1977.

(Accepted for publication August 16, 1977.) 